\title{
Review Article \\ Thyroid Peroxidase Antibody and Screening for Postpartum Thyroid Dysfunction
}

\author{
Mohamed A. Adlan ${ }^{1}$ and Lakdasa D. Premawardhana ${ }^{1,2}$ \\ ${ }^{1}$ Section of Endocrinology and Diabetes, Department of Medicine, Caerphilly District Miners Hospital, \\ St. Martin's Road, Caerphilly CF83 2WW, UK \\ ${ }^{2}$ C2 Link, University Hospital of Wales, Heath Park, Cardiff CF14 4XN, UK
}

Correspondence should be addressed to Lakdasa D. Premawardhana, premawardhanald@cardiff.ac.uk

Received 14 March 2011; Accepted 10 May 2011

Academic Editor: Kris Gustave Poppe

Copyright ( 2011 M. A. Adlan and L. D. Premawardhana. This is an open access article distributed under the Creative Commons Attribution License, which permits unrestricted use, distribution, and reproduction in any medium, provided the original work is properly cited.

\begin{abstract}
Postpartum thyroid dysfunction (PPTD) is a common disorder which causes considerable morbidity in affected women. The availability of effective treatment for hypothyroid PPTD, the occurrence of the disease in subsequent pregnancies and the need to identify subjects who develop long term hypothyroidism, has prompted discussion about screening for this disorder. There is currently no consensus about screening as investigations hitherto have been variable in their design, definitions and assay frequency and methodology. There is also a lack of consensus about a suitable screening tool although thyroid peroxidase antibody (TPOAb) is a leading contender. We present data about the use of TPOAb in early pregnancy and its value as a screening tool. Although its positive predictive value is moderate, its sensitivity and specificity when used in early pregnancy are comparable or better compared to other times during pregnancy and the postpartum period. Recent studies have also confirmed this strategy to be cost effective and to compare favourably with other screening strategies. We also explore the advantages of universal screening.
\end{abstract}

\section{Postpartum Thyroid Dysfunction Is Common}

Postpartum thyroid dysfunction (PPTD) is a common disorder which causes considerable morbidity in some women [1]. The availability of effective treatment particularly for the symptomatic hypothyroid phase, and the awareness that hypothyroidism is a long-term consequence in a significant minority of these subjects, has prompted discussion about screening for PPTD $[2,3]$. However, there is currently no consensus because of unresolved issues about an effective and sensitive prediction tool.

The current worldwide pooled prevalence for PPTD is estimated to be $8 \%(95 \%$ CI $7.8-8.2 \%)$, with regional variations-USA 5.7\%, Asia 4.4\%, Spain 9.3\%, Sweden $7.3 \%$, and The Netherlands 6.3\% [4]. These differences may be due to (a) variable study design (timing and number of thyroid tests), (b) definition of PPTD, (c) prevalence of thyroid peroxidase antibody (TPOAb), (d) assay methods used (antimicrosomal versus TPOAb), and (e) population characteristics (including prevalence of type 1 diabetes mellitus (T1DM), PPTD in previous pregnancies, iodine intake and genetics) [5].

The early hyperthyroid phase of PPTD causes minimal symptoms and hardly ever requires specific treatment. However, the hypothyroid phase which occurs later often needs to be treated with thyroxine for up to 9 months [6]. A significant number of subjects who have hypothyroid PPTD remain so at the end of the first postpartum year and require long-term thyroxine replacement [7-10].

\section{The Rationale for Screening for PPTD}

There are strong proponents on both sides of the thyroid screening in pregnancy debate $[3,11,12]$. The Endocrine Society currently recommends selective screening of highrisk individuals only [13]. However, Vaidya et al. elegantly demonstrated that screening of high-risk individuals alone would miss $30 \%$ of hypothyroid and $69 \%$ of hyperthyroid pregnant women [14]. A recent randomised controlled trial 
[15] and another study [16] confirmed this initial impression about selective screening.

Those who advocate screening for PPTD cite the following reasons in support of their argument:

(a) avoiding morbidity associated particularly with hypothyroid PPTD,

(b) predicting the need for long-term thyroxine treatment at the end of the first postpartum year,

(c) identifying subjects who might develop PPTD in subsequent pregnancies,

(d) identifying subjects for followup to detect long-term hypothyroidism several years from initial diagnosis $[10,17]$.

\section{The Role of TPOAb in Pregnancy-Associated Morbidity}

The prevalence of microsomal and TPOAb in pregnant women varies between 2.8 and $19.6 \%$ worldwide [18], and a recent pooled prevalence from published data was estimated to be 15.3-16\% [4]. Although its role in causing PPTD remains speculative, the presence of TPOAb identifies a subset of pregnant women who have a higher risk of developing PPTD $[4,19]$. In addition, TPOAb also identifies women who have a higher risk of long-term hypothyroidism after PPTD [10, 17, 20, 21].

The presence of TPOAb during pregnancy is also associated with miscarriage and preterm delivery [6], an IQ decrement in children of even euthyroid mothers [22], and postpartum psychiatric morbidity $[23,24]$. The mechanisms for these remain unclear.

\section{A Screening Tool for PPTD}

The clinical utility of a screening tool depends on several factors. It needs to be sensitive with a high positive predictive value (to detect every subject with the condition if possible), easy to perform (using uncomplicated technology), cheap (so that widespread use is cost-beneficial), and harmless to the subject tested.

\section{TPOAb as a Screening Tool}

Some authorities recommend TPOAb as a suitable screening tool for PPTD $[4,25]$. The relative high prevalence of TPOAb in early pregnancy, its easy measurement with current assays, and its role as a risk marker for PPTD and long-term hypothyroidism have been cited in its favour as discussed above. Although earlier assays measured microsomal antibodies, more recent assays measure antibodies to its specific antigen, thyroid peroxidase, that is, TPOAb. Sensitive assays for TPOAb which are relatively cheap to perform are now available commercially and are in use worldwide [26, 27].

\subsection{TPOAb and PPTD}

5.1.1. Noninterventional Studies. An extensive analysis of published data by Nicholson et al. examined the prevalence of TPOAb and the risk of developing PPTD in these subjects. They estimated a pooled prevalence of TPOAb of $16.2 \%$ and a risk ratio of 5.7 for the development of PPTD (Table 1) [4].

A closer examination of these data helps us understand why it is difficult to reach a consensus about the use of TPOAb for screening, despite favourable epidemiological and statistical evidence.

(i) Several of these studies measured antimicrosomal antibodies as this was acceptable practice at the time. However, following the identification of thyroid peroxidase (TPO) as the incriminating antigen, more recent studies have used a TPOAb assay.

(ii) Furthermore, these antibodies were tested at variable points during pregnancy and postpartum. We contend that, for a prediction tool to be useful, it needs to be used well in advance of the predicted event that is, early pregnancy in this instance. Although the risk ratios appear to be higher when postpartum testing is done, we feel an early antepartum test would be more pragmatic (Table 2). The immune modulation of pregnancy and its effect on TPOAb (significant decrease in levels as pregnancy advances and a rebound increase postpartum) need to be understood and taken into account.

(iii) The definitions of PPTD were variable in these studies.

(iv) There is also a significant variability of the populations studied. There may well have been a difference in factors known to increase the risk of PPTD which were not uniformly reported-iodine intake, genetic predisposition to thyroid disease, demographic and behavioural characteristics (age, parity, smoking, gender of offspring, etc.), prevalence of type 1 diabetes mellitus, a history of PPTD in previous pregnancies, and a family history of thyroid disease.

(v) There is no clear indication of the length of followup in these studies either-raising the possibility of missing some PPTD subjects.

Although extrapolating from these studies to a general population is not wise, we do not feel that the utility of TPOAb, when used appropriately as a screening tool, is significantly diminished.

Since then, other investigators have also studied this relationship. Diaz et al. found a 44\% prevalence of TPOAb in 25 subjects (out of 157) who developed PPTD [28]. Only $4.5 \%$ had TPOAb in the group who did not develop PPTD $(P=.001)$. Filippi et al. found a combined prevalence of TPOAb and antithyroglobulin antibodies (TgAb) of $9.3 \%$ and $28 / 43(65.1 \%)$ had developed thyroid dysfunction at the end of the first year [29].

An earlier analysis of published data has shown that the sensitivity, specificity, and positive predictive value (PPV) of 
TABLE 1: TPOAb prevalence and the risk of developing PPTD (adapted from [4] with permission).

\begin{tabular}{|c|c|c|c|c|}
\hline $\begin{array}{l}\text { Author (number of } \\
\text { subjects in study) }\end{array}$ & $\begin{array}{c}\text { Number } \% \text { prevalence of } \\
\text { TPOAb }(95 \% \text { CI })\end{array}$ & $\begin{array}{l}\text { Number with } \\
\text { PPTD }\end{array}$ & $\begin{array}{l}\text { TPOAb +ve } \\
\text { with PPTD }\end{array}$ & $\begin{array}{l}\text { Risk ratio for PPTD in TPOAb } \\
\text { +ve subjects }(95 \% \mathrm{CI})\end{array}$ \\
\hline Shahbazian (1040) & $248 / 24(21-26)$ & 119 & 73 & $5(4-7)$ \\
\hline Kent (718) & $86 / 12(10-14)$ & 86 & 55 & $13(9-19)$ \\
\hline Furlanetto (284) & $13 / 5(3-8)$ & 12 & 2 & $4(0.9-15)$ \\
\hline Lucas (579) & No information & 45 & 30 & - \\
\hline Kuijpens (291) & $31 / 14(11-19)$ & 15 & 10 & $17(6-46)$ \\
\hline Lervang (591) & $38 / 6(5-9)$ & 23 & 20 & $97(30-312)$ \\
\hline Jansson (460) & $44 / 10(7-13)$ & 30 & 23 & $31(14-68)$ \\
\hline Dahlberg (224) & $11 / 4.9(2.1-7.7)$ & 12 & 11 & $58(18.2-184.9)$ \\
\hline Amino (507) & $62 / 12(10-15)$ & 28 & 25 & $59(18.6-192)$ \\
\hline Totals (4914) & 586 & 419 & 279 & \\
\hline Pooled estimate & $16.2 \%(16-16.4)$ & & & $5.7(5.3-6.3)$ \\
\hline
\end{tabular}

TABLE 2: TPOAb and its utility for screening for PPTD: timing of screening and sensitivity, specificity, and PPV (adopted from [25] with permission).

\begin{tabular}{lccc}
\hline Time of screening & Sensitivity & Specificity & PPV \\
\hline Early pregnancy & $0.67-1$ & $0.62-0.93$ & $0.31-0.55$ \\
3rd trimester & 0.71 & 0.92 & 0.52 \\
Delivery/early postpartum & $0.45-0.89$ & $0.91-0.97$ & $0.4-0.73$ \\
Late postpartum & $0.46-0.86$ & $0.9-0.98$ & $0.53-0.78$ \\
\hline
\end{tabular}

TPOAb as a prediction tool are widely variable and depend on the time of testing amongst other factors (Table 2) [25].

5.1.2. Interventional Studies. Several interventional studies also have strengthened our knowledge about the role of TPOAb in morbidity associated with pregnancy and PPT. In one study, the administration of thyroxine to euthyroid women with TPOAb significantly reduced the risk of miscarriage [30]. A more recent well designed, randomised placebo-controlled study elegantly showed that giving selenium to pregnant women significantly reduced postpartum TPOAb titres and the incidence of PPTD [31]. These effects of selenium may be mediated through selenoenzymes which are abundant in the thyroid gland [32]. Although these studies do not prove a causative role, they further clarify the relationship of TPOAb to pregnancy outcomes and PPTD.

\subsection{TPOAb and Long-Term Thyroid Dysfunction after PPTD.} The presence of TPOAb in pregnancy is also a marker for long-term thyroid dysfunction [5]. A recently published 12year follow-up study comparing women who had PPTD with controls found odds ratios for the risk of hypothyroidism of 4.8 for PPTD, 9.7 for hypothyroid PPTD, and 51.4 for TPOAb in association with hypothyroid PPTD [10]. This increased risk is broadly in line with 2 previous studies in which TPOAb-positive women who had PPTD were followed up for a mean period of 60 and 78 months. These investigators found hypothyroidism in 26\% [33] and $46 \%$ [17], respectively, which were significantly higher in comparison to TPOAb negative controls. The relationship of the hypothyroid phase of PPTD to the development of longterm hypothyroidism cannot be over-emphasized, and this is a consistent feature of studies published so far.

5.3. Timing of Screening. Although PPV remains only moderately high, early pregnancy (first trimester) screening seems to confer the highest sensitivity and specificity [25]. Early pregnancy screening would seem highly pragmatic in a screening programme too.

5.4. Cost Benefit. A recent study found screening pregnant women in the first trimester with TPOAb to be cost-effective and to compare favourably with other screening strategies currently in use [34]. These data supported previous evidence from other investigators [35].

However, there are two objections to using TPOAb as a screening tool based on the current data.

(a) No consensus can be achieved of its utility in screening because of the heterogeneity of the reported studies-opponents of screening often quote the difficulty in interpreting and extrapolating results of published studies because of a high degree of variability (see comments above).

(b) The occurrence of PPTD in antibody-negative subjects also detracts from its value as a screening tool-there are several studies where PPTD has been reported in TPOAb-(or microsomal antibody) negative subjects $[10,36,37]$. The reasons for this are speculative-insensitivity of assays used to detect TPOAb, the presence of TgAb only, sampling time during pregnancy, population characteristics, and so forth.

\section{TPOAb Combined with Clinical Data}

Some investigators have studied the utility of TPOAb combined with clinical features in predicting PPTD. Da Costa et al. recruited 98 unselected women at 9-12 weeks of pregnancy and followed them up for 1 year after delivery. 
TPOAb was present in 10.2\% (95\% CI 4.1-16.3\%) and PPTD developed in $10.2 \%$ (95\% CI 4.1-16.3\%). A goitre in early pregnancy and TPOAb were significantly associated with PPTD $(P=.01, .001$, resp.) [38]. The sensitivity $(60 \%, 95 \%$ CI 31.3-83.2) and specificity (95.5\%, 95\% CI 88.9-98.2\%) compared with data reported in earlier studies (Table 2). But the PPV increased from 60 to $82.4 \%$ when goitre and family history of thyroid disease were added to TPOAb as risk factors. Others have indicated the importance of ultrasound echogenicity in predicting long-term thyroid dysfunction following PPTD [17], and the importance of other antenatal indices needs to be confirmed and their clinical utility determined $[39,40]$.

\section{Conclusions}

The question of screening for PPTD is intimately linked with the broader issue of screening for thyroid dysfunction in pregnancy [41]. We have concentrated on the former for this review. Experts and expert societies take a variable stance on the question of screening in pregnancy, but there is broad consensus about its advantages. Most advocate targeted screening but numerous studies have indicated the futility of this approach as a significant number of affected women with thyroid dysfunction will fail to be identified [14-16].

PPTD is a common condition causing significant morbidity. Screening for PPTD is safe and has many benefits and few disadvantages (maternal anxiety is mentioned frequently in this regard). Its early detection may improve quality of life and reduce morbidity in subsequent pregnancies and in the long term. Cost benefit analyses have demonstrated definite cost advantages in screening.

TPOAb is a good screening tool and has been examined by many investigators for predicting PPTD. Although there are limited data of the benefits of intervention in TPOAbpositive women in early pregnancy $[30,31]$, such a paucity of data should not deter us from recommending a screening strategy using it. TPOAb in early pregnancy (first trimester) using a currently available sensitive assay seems to be the best available tool at present despite all its imperfections. What about women who are TPOAb negative but still develop PPTD? There is no clear explanation for PPTD in these women but this should not prevent clinicians from adopting a screening strategy using TPOAb where the majority who would develop PPTD would be identified.

The question about targeted or universal screening continues to be debated. Given the current evidence of the ineffectiveness of targeted screening in identifying all women with thyroid dysfunction during pregnancy and the lack of clarity of expert advice, we would advocate universal screening. We believe adopting such a stance could only be useful and its benefits could then be examined in future studies.

\section{Acknowledgment}

The authors thank Professor John Lazarus for his continuing inspiration and guidance.

\section{References}

[1] J. H. Lazarus, "Clinical manifestations of postpartum thyroid disease," Thyroid, vol. 9, no. 7, pp. 685-689, 1999.

[2] J. H. Lazarus, "Prediction of postpartum thyroiditis," European Journal of Endocrinology, vol. 139, no. 1, pp. 12-13, 1998.

[3] N. Amino, H. Tada, Y. Hidaka, L. M. Crapo, and A. StagnaroGreen, "Therapeutic controversy. Screening for postpartum thyroiditis," Journal of Clinical Endocrinology and Metabolism, vol. 84, no. 6, pp. 1813-1821, 1999.

[4] W. K. Nicholson, K. A. Robinson, R. C. Smallridge, P. W. Ladenson, and N. R. Powe, "Prevalence of postpartum thyroid dysfunction: a quantitative review," Thyroid, vol. 16, no. 6, pp. 573-582, 2006.

[5] J. H. Lazarus, "The continuing saga of postpartum thyroiditis," Journal of Clinical Endocrinology and Metabolism, vol. 96, no. 3, pp. 614-616, 2011.

[6] A. Stagnaro-Green, "Postpartum thyroiditis," Best Practice \& Research Clinical Endocrinology \& Metabolism, vol. 18, pp. 305-308, 2004.

[7] H. Y. M. Fung, M. Kologlu, K. Collison et al., "Postpartum thyroid dysfunction in Mid Glamorgan," British Medical Journal, vol. 296, no. 6617, pp. 241-244, 1988.

[8] A. F. Muller, H. A. Drexhage, and A. Berghout, "Postpartum thyroiditis and autoimmune thyroiditis in women of child bearing age: recent insights and consequences for antenatal and postnatal care," Endocrine Reviews, vol. 22, pp. 605-630, 2001.

[9] B. G. A. Stuckey, G. Neil Kent, and J. R. Allen, “The biochemical and clinical course of postpartum thyroid dysfunction: the treatment decision," Clinical Endocrinology, vol. 54, no. 3, pp. 377-383, 2001.

[10] B. G. A. Stuckey, G. N. Kent, L. C. Ward, S. J. Brown, and J. P. Walsh, "Postpartum thyroid dysfunction and the long-term risk of hypothyroidism: results from a 12-year follow-up study of women with and without postpartum thyroid dysfunction," Clinical Endocrinology, vol. 73, no. 3, pp. 389-395, 2010.

[11] T. F. Davies, "The ATA, the endocrine society, and AACE confuse endocrinologists on thyroid disease in pregnancy," Thyroid, vol. 10, no. 2, p. 107, 2000.

[12] American College of Obstetrics and Gynecology, "Thyroid disease in pregnancy," International Journal of Gynecology \& Obstetrics, vol. 79, pp. 171-180, 2002.

[13] M. Abalovich, N. Amino, L. A. Barbour et al., "Management of thyroid dysfunction during pregnancy and postpartum: an Endocrine Society Clinical Practice Guideline," The Journal of Clinical Endocrinology and Metabolism, vol. 92, 8, pp. S1-S47, 2007.

[14] B. Vaidya, S. Anthony, M. Bilous et al., "Brief report: detection of thyroid dysfunction in early pregnancy: universal screening or targeted high-risk case finding?" Journal of Clinical Endocrinology and Metabolism, vol. 92, no. 1, pp. 203-207, 2007.

[15] R. Negro, A. Schwartz, R. Gismondi, A. Tinelli, T. Mangieri, and A. Stagnaro-Green, "Universal screening versus case finding for detection and treatment of thyroid hormonal dysfunction during pregnancy," Journal of Clinical Endocrinology and Metabolism, vol. 95, no. 4, pp. 1699-1707, 2010.

[16] J. Horacek, S. Spitalnikova, B. Dlabalova et al., "Universal screening detects two-times more thyroid disorders in early pregnancy than targeted high-risk case finding," European Journal of Endocrinology, vol. 163, no. 4, pp. 645-650, 2010. 
[17] L. D. K. E. Premawardhana, A. B. Parkes, F. Ammari et al., "Postpartum thyroiditis and long-term thyroid status: prognostic influence of thyroid peroxidase antibodies and ultrasound echogenicity," Journal of Clinical Endocrinology and Metabolism, vol. 85, no. 1, pp. 71-75, 2000.

[18] R. C. Smallridge, "Postpartum thyroid disease: a model of immunologic dysfunction," Clinical and Applied Immunology Reviews, vol. 1, no. 2, pp. 89-103, 2001.

[19] B. Harris, S. Othman, J. A. Davies et al., "Association between postpartum thyroid dysfunction and thyroid antibodies and depression," British Medical Journal, vol. 305, no. 6846, pp. 152-156, 1992.

[20] F. Azizi, "The occurence of permanent thyroid failure in patients with subclinical postpartum thyroiditis," European Journal of Endocrinology, vol. 153, no. 3, pp. 367-371, 2005.

[21] A. Lucas, E. Pizarro, M. L. Granada, I. Salinas, J. Roca, and A. Sanmartí, "Postpartum thyroiditis: long-term follow-up," Thyroid, vol. 15, no. 10, pp. 1177-1181, 2005.

[22] V. J. Pop, E. De Vries, A. L. Van Baar et al., "Maternal thyroid peroxidase antibodies during pregnancy: a marker of impaired child development?" Journal of Clinical Endocrinology and Metabolism, vol. 80, no. 12, pp. 3561-3566, 1995.

[23] V. J. M. Pop, H. A. M. De Rooy, H. L. Vader, D. Van Der Heide, M. M. Van Son, and I. H. Komproe, "Microsomal antibodies during gestation in relation to postpartum thyroid dysfunction and depression," Acta Endocrinologica, vol. 129, no. 1, pp. 26-30, 1993.

[24] V. J. Pop, H. A. de Rooy, H. L. Vader et al., "Postpartum thyroid dysfunction and depression in an unselected population," New England Journal of Medicine, vol. 324, no. 25, pp. 1815-1816, 1991.

[25] J. H. Lazarus and L. D. K. E. Premawardhana, "Screening for thyroid disease in pregnancy," Journal of Clinical Pathology, vol. 58, no. 5, pp. 449-452, 2005.

[26] C. J. Groves, R. D. Howells, S. Williams, C. Darke, and A. B. Parkes, "Primary standardization for the ELISA of serum thyroperoxidase and thyroglobulin antibodies and their prevalence in a normal Welsh population," Journal of Clinical and Laboratory Immunology, vol. 32, no. 3, pp. 147-151, 1990.

[27] P. Laurberg, K. M. Pedersen, E. Vittinghus, and S. Ekelund, "Sensitive enzyme-linked immunosorbent assay for measurement of autoantibodies to human thyroid peroxidase," Scandinavian Journal of Clinical and Laboratory Investigation, vol. 52, no. 7, pp. 663-669, 1992.

[28] J. I. J. Díaz, F. L. De Castro, B. C. García, F. S. Balduz, J. S. Marcos, and C. M. Dal Gesso, "Incidence of postpartum thyroiditis and study of possible associated factors," Medicina Clinica, vol. 132, no. 15, pp. 569-573, 2009.

[29] U. Filippi, R. Brizzolara, D. Venuti et al., "Prevalence of postpartum thyroiditis in Liguria: an observational study," Journal of Endocrinological Investigation, vol. 31, no. 12, pp. 10631068, 2008.

[30] R. Negro, G. Formoso, T. Mangieri, A. Pezzarossa, D. Dazzi, and H. Hassan, "Levothyroxine treatment in euthyroid pregnant women with autoimmune thyroid disease: effects on obstetrical complications," Journal of Clinical Endocrinology and Metabolism, vol. 91, no. 7, pp. 2587-2591, 2006.

[31] R. Negro, G. Greco, T. Mangieri, A. Pezzarossa, D. Dazzi, and H. Hassan, "The influence of selenium supplementation on postpartum thyroid status in pregnant women with thyroid peroxidase autoantibodies," Journal of Clinical Endocrinology and Metabolism, vol. 92, no. 4, pp. 1263-1268, 2007.
[32] J. Köhrle, F. Jakob, B. Contempré, and J. E. Dumont, "Selenium, the thyroid, and the endocrine system," Endocrine Reviews, vol. 26, no. 7, pp. 944-984, 2005.

[33] R. Jansson, P. A. Dahlberg, and F. A. Karlsson, "Postpartum thyroiditis," Bailliere's Clinical Endocrinology and Metabolism, vol. 2, no. 3, pp. 619-635, 1988.

[34] C. Dosiou, G. D. Sanders, S. S. Araki, and L. M. Crapo, "Screening pregnant women for autoimmune thyroid disease: a cost-effectiveness analysis," European Journal of Endocrinology, vol. 158, no. 6, pp. 841-851, 2008.

[35] D. E. Bonds and K. A. Freedberg, "Cost-effectiveness of prenatal screening for postpartum thyroiditis," Journal of Women's Health and Gender-Based Medicine, vol. 10, no. 7, pp. 649-658, 2001.

[36] A. Stagnaro-Green, A. Schwartz, R. Gismondi, A. Tinelli, T. Mangieri, and R. Negro, "High rate of persistent hypothyroidism in a large-scale prospective study of postpartum thyroiditis in Southern Italy," Journal of Clinical Endocrinology and Metabolism, vol. 96, no. 3, pp. 652-657, 2011.

[37] J. L. Kuijpens, M. De Haan-Meulman, H. L. Vader, V. J. Pop, W. M. Wiersinga, and H. A. Drexhage, "Cell-mediated immunity and postpartum thyroid dysfunction: a possibility for the prediction of disease?" Journal of Clinical Endocrinology and Metabolism, vol. 83, no. 6, pp. 1959-1966, 1998.

[38] S. Mamede Da Costa, L. Sieiro Netto, C. M. Coeli, A. Buescu, and M. Vaisman, "Value of combined clinical information and thyroid peroxidase antibodies in pregnancy for the prediction of postpartum thyroid dysfunction," American Journal of Reproductive Immunology, vol. 58, no. 4, pp. 344-349, 2007.

[39] A. A. Kokandi, A. B. Parkes, L. D. K. E. Premawardhana, R. John, and J. H. Lazarus, "Association of postpartum thyroid dysfunction with antepartum hormonal and immunological changes," Journal of Clinical Endocrinology and Metabolism, vol. 88, no. 3, pp. 1126-1132, 2003.

[40] G. Mazziotti, A. B. Parkes, M. Lage, L. D. K. E. Premawardhana, F. F. Casanueva, and J. H. Lazarus, "High leptin levels in women developing postpartum thyroiditis," Clinical Endocrinology, vol. 60, no. 2, pp. 208-213, 2004.

[41] D. Glinoer and C. A. Spencer, "Serum TSH determinations in pregnancy: how, when and why?" Nature Reviews Endocrinology, vol. 6, no. 9, pp. 526-529, 2010. 


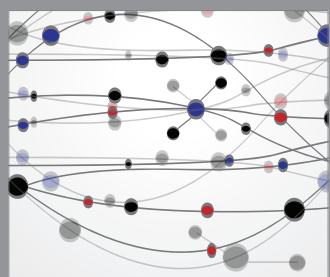

The Scientific World Journal
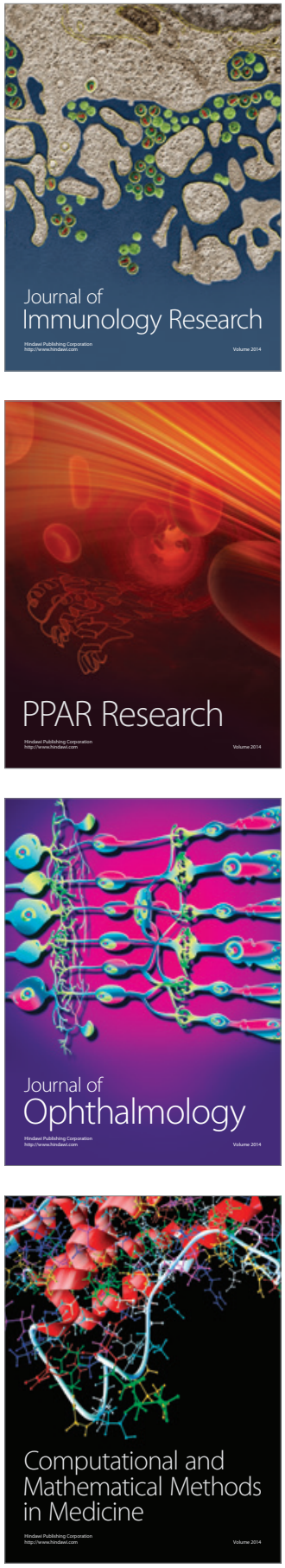

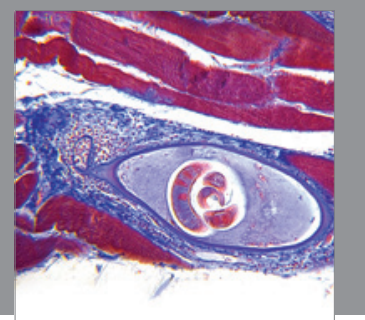

Gastroenterology

Research and Practice
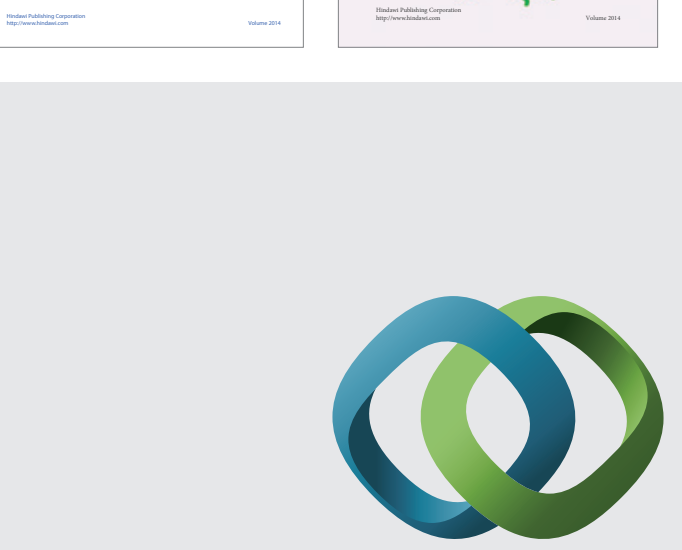

\section{Hindawi}

Submit your manuscripts at

http://www.hindawi.com
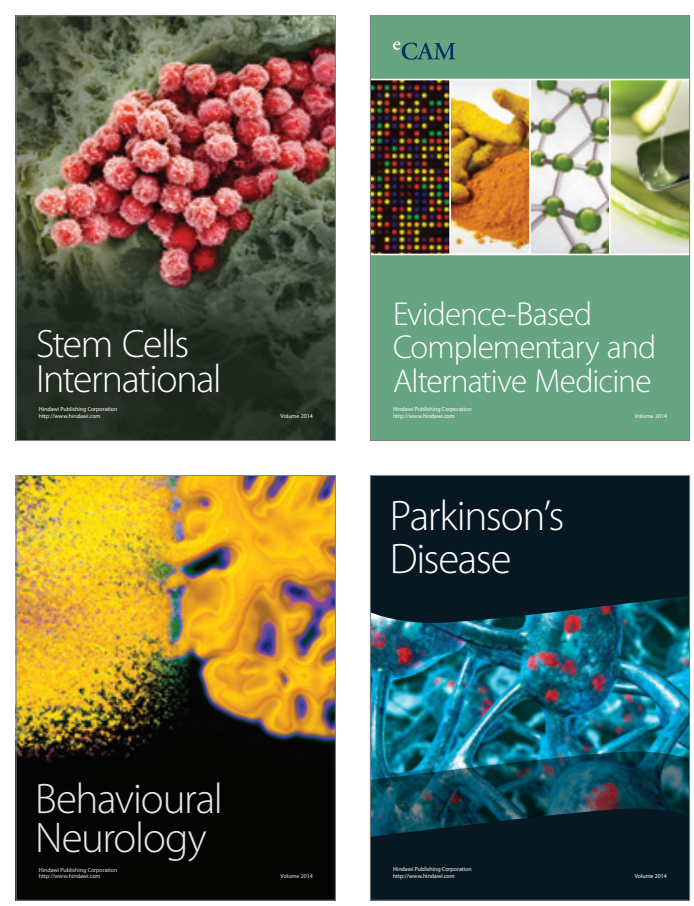

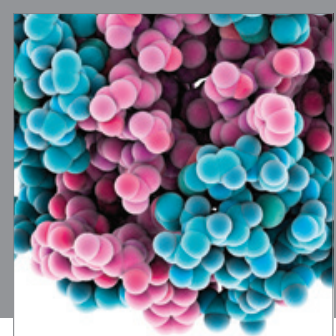

Journal of
Diabetes Research

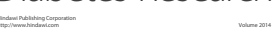

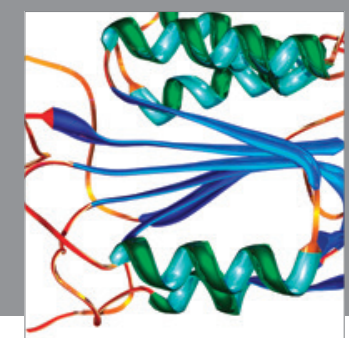

Disease Markers
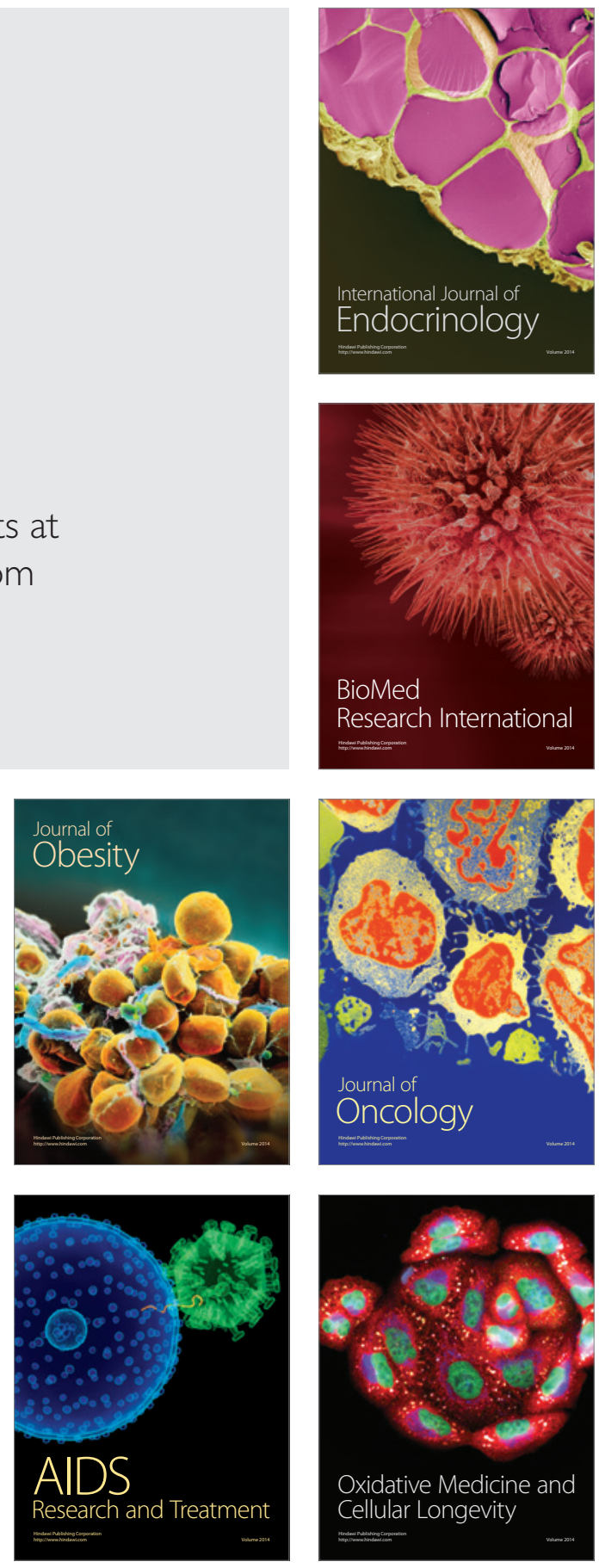